\title{
LA ENSEÑANZA DE LAS METODOLOGÍAS EN LAS CIENCIAS SOCIALES EN BRASIL
}

\section{BRAZIL SOCIAL SCIENCES METHODOLOGY TEACHING}

Dr. Alfredo A. Gugliano (alfredogugliano@hotmail.com) Departamento de Ciencia Política. Universidade Federal do Rio Grande do Sul (Porto Alegre, Brasil)

Dr. Pedro Robertt (probertt21@gmail.com) Instituto de Sociología y Política. Universidade Federal de Pelotas (Pelotas, Brasil)

\section{Abstract}

The present article focuses on the concern to know how the process of preparing investigators at Brazilian universities is being developed. One of the first difficulties being dealt with involves the predominant of segmented approach of the scientific investigation. This leads to the questioning of methodologies used to prepare these investigators. Among other several difficulties, the epistemological aspects up to the methodological ideas on the preparation of investigators are included. The structures of the subjects, their relation to the methodologies and the relations between methodological teaching and investigation practices are questioned. The preparation of critical social scientists in which extremely rigid different separations from the preparation processes is at stake here.

Key words: methodology, teaching, investigation, university, social scientists

\section{Resumen}

El artículo parte de la preocupación de saber cómo se está desarrollando la formación de investigadores en las universidades brasileñas. Una primera dificultad que se coloca es el predominio de un abordaje segmentado de la investigación científica. Eso lleva a un cuestionamiento sobre si particularmente las asignaturas de metodologías sirven para formar investigadores. Entre diversas dificultades, se incluyen desde aspectos epistemológicos hasta los impactos de las asignaturas metodológicas sobre la formación de los investigadores. Se cuestionan las estructuras de las disciplinas, su relación con las metodologías, y las relaciones entre enseñanza metodológica y prácticas de investigación. Lo que está en juego es la formación de científicos sociales críticos en que se cuestionen diferentes separaciones extremamente rígidas de los procesos de formación.

Palabras clave: metodología, enseñanza, investigación, universidad, científicos sociales 


\section{Introducción}

En Aprendiendo a trabajar, Willis (1988) muestra cómo, en la sociedad inglesa, las juventudes de las clases trabajadoras se autocondenan a desarrollar tareas profesionales propias de su clase. Partiendo de un análisis etnográfico entre jóvenes que presentan resistencia a la cultura escolar, demuestra la incapacidad que tienen las escuelas oficiales de representar una posibilidad real de ascensión social para los hijos de los segmentos más pobres de la población.

Este es un proceso complejo que articula no solamente la estructura de las instituciones educacionales, sino también la selección de conocimientos y la forma como estos son enseñados. Así se comprende porque Bourdieu y Passeron (1982) denominaron violencia simbólica de la acción pedagógica a una educación que es transmitida no sólo a través de la selección de contenidos, sino también mediante la manera cómo estos son presentados. Se trata de la forma cómo, en situaciones específicas, los valores de sectores o clases dominantes son desanclados de su especificidad de origen y presentados como universales.

Inspirados en esta discusión, una temática que consideramos relevante trata de la didáctica de la enseñanza de metodologías en las Ciencias Sociales. Más específicamente, nos preocupa cómo se está desarrollando la formación de investigadores sociales en las universidades brasileñas y su relación con la mencionada asignatura.

Como es conocido, los orígenes de las Ciencias Sociales fueron marcados por el intento de equiparación con las Ciencias Naturales. Esto mediante una visión idealizada de lo que sería la ciencia, considerada como un conocimiento completamente racional y con procedimientos ejecutados por técnicos altamente calificados en inexpugnables laboratorios científicos. Al método científico cabría un lugar privilegiado en este mundo, el papel de superar a un sentido común en el cuál estaba ahogada buena parte de la humanidad (1).

En esta concepción, enseñar metodologías equivaldría a realizar ceremoniales de iniciación en los cuáles algunos pocos escogidos recibirían instrumentos por los cuales ascenderían a un nivel superior de comprensión de la realidad. Así como en las escuelas donde algunos aprenden a ser la élite y otros no.

Hasta qué punto una visión elitista de ciencia acabó interfiriendo en el proceso de formación de científicos sociales en Brasil, es el telón de fondo que nos lleva a discutir la enseñanza de las metodologías en nuestro campo de conocimiento. Con esto pretendemos contribuir a la ampliación del debate sobre la necesidad de repensar esta cuestión y fortalecer la formación investigativa en nuestra región.

\section{La formación investigativa y las asignaturas de metodología}

Cuando se habla en formación de investigadores una pregunta que puede servir de guía es: ¿cómo ocurre el acercamiento de los estudiantes a las tareas de investigación? Sobre este tema Bastos (2006) intenta reconstruir la trayectoria de importantes sociólogos brasileños, e indica cómo pueden ocurrir distintas aproximaciones a partir de las cuales jóvenes estudiantes empiezan a enamorarse de la carrera de científico 
social, sea por influencia de colegas de clase, incorporación a grupos de trabajo de docentes o hasta por cuestiones familiares o de fondo ideológico, entre otras.

Un punto en común en medio a posibles diferentes trayectorias es el papel de la universidad en ese proceso. Como afirma Melucci (2005), la capacidad de auto-reflexión sobre la realidad no es una exclusividad de las universidades, no obstante, principalmente en estas instituciones -como también en agencias privadas o públicas de investigación- los investigadores poseen recursos institucionales para reflexionar sobre las prácticas de la sociedad.

Pero, ¿cuándo y bajo qué características ocurre la introducción de los jóvenes en el universo de la investigación en el interior de los cursos de ciencias sociales? Las asignaturas de metodología, sin duda, representan un espacio importante para la inserción en el mundo de la exploración científica. Sin embargo, una primera dificultad para que cumplan con esta tarea reside en el predominio de un abordaje segmentado de los diferentes momentos del proceso de investigación: de cuestiones epistemológicas o dirigidas hacia grandes objetivos e hipótesis de investigación; de diseño de investigación, de trabajo de campo o de aplicación de técnicas (divididas generalmente entre las de tipo cuantitativo y cualitativo); o, finalmente, de análisis de resultados (2).

Además, puede cuestionarse que las asignaturas de metodologías, en la formación de investigadores sociales, pierden gran parte de su funcionalidad cuando aprisionan el proceso de elaboración científica en la misma jaula donde la concepción "bancaria” de educación deja cerrado al conocimiento en general. Es así como Freire (1981) denominó al modo como el proceso de aprendizaje es desarrollado, de forma que el docente "deposita" -como en los Bancos- el conocimiento en la cabeza de estudiantes, condenados a memorizar y repetir. En nuestro caso, lo que se deposita son "métodos y/o técnicas".

De esta manera, los alumnos son expuestos a una carga considerable de conocimientos abstractos relacionados con "las metodologías", pero pocos saben lo que hacer con lo aprendido, hecho que deja una enorme complicación en las manos de los docentes de metodología, porque si esta asignatura no sirve para formar investigadores ¿para qué estamos enseñando métodos? Ese, además, no es un problema sólo de las Ciencias Sociales. Si analizamos la literatura sobre el tema en el área más general de las Humanidades veremos que de las Artes al Derecho; de la Sociología a la Historia; o de la Filosofía a la Ciencia Política; existen cuestionamientos sobre la enseñanza de las metodologías (Betancourt 1990, Cardoso 1986).

Un espacio más reciente de formación de investigadores sociales es representado por los postgrados en ciencias sociales. A nivel internacional, los programas de maestrías y doctorados representan espacios privilegiados para el desarrollo de investigaciones de alto nivel y, en consecuencia, para la formación de investigadores.

La dificultad inicial que puede presentarse en estos programas es el riesgo de terminar ofreciendo más de lo mismo, reproduciendo esquemas pedagógicos propuestos en las formaciones de grado, es decir, etapas monótona y burocráticamente estipuladas, con concepciones dominantes de "depósito" de conocimientos" y con docentes individualmente responsables en forma a veces quijotesca de la formación científica de los estudiantes 
Además, al restringirse la formación investigativa de alta calidad a los postgrados, se acaba reproduciendo una concepción elitista de la ciencia, en la cual el conocimiento queda en las manos apenas de los segmentos sociales capaces de alcanzar los escalones más altos del sistema educacional.

En ese sentido, una tarea que quizás se presente como urgente sea la de empezar a rediscutir adónde pretendemos llegar con nuestras clases metodológicas.

\section{Sobre los objetivos de la enseñanza de las metodologías}

Una parte considerable de los textos brasileños sobre el desarrollo de las ciencias sociales sugiere que las principales cuestiones que hoy discutimos en el campo de la enseñanza de las metodologías no son problemas aislados, estando presentes en casi todas las universidades el país. Son dificultades relacionadas con aspectos epistemológicos, que corresponden a las formas de utilización de las diferentes perspectivas metodológicas, así como con los resultados del proceso educativo y los impactos de las asignaturas metodológicas sobre los estudiantes (Bomeny y Birman 1991, Pessanha y Villas Bôas 1995, Reis, Reis y Velho 1997).

En lo relacionado a la didáctica de las metodologías de la investigación un elemento que se destaca, en prácticamente todos los cursos de grado, es la atribución exclusiva de enseñar métodos y/o técnicas de investigación conferida a las asignaturas que tratan del tema (3).

Si analizamos, por ejemplo, los programas académicos de buena parte de los cursos de ciencias sociales brasileños, notaremos que las fronteras científicas son muy bien delimitadas, existiendo asignaturas en las cuales se estudian los métodos (¡las metodologías!), otras en las cuales se practican los métodos aprendidos (ilas prácticas de investigación!) y, finalmente, aquellas asignaturas en las cuales se discuten las teorías (¡las teóricas!).

Desde esta perspectiva de "enseñar métodos y/o técnicas" es que buena parte de los docentes enfrentan el desafío de presentar un conjunto de procedimientos que solamente cobran sentido como herramientas de investigación científica, en la confrontación de categorías de análisis con aquello que convenimos en llamar de realidad empírica.

La separación de la enseñanza de los métodos del análisis de los procesos de investigación, aun siendo hegemónica, no deja de ser problemática. En un sentido general, desde diferentes tradiciones de pensamiento, algunos autores cuestionaron las separaciones rígidas entre teorías, metodologías, procesos de investigación y realidades empíricas. Así, por ejemplo, August Comte, quien dio nombre a la sociología a mediados del siglo XIX, alertaba sobre la incorrección de pensar en metodologías desvinculadas de su empleo práctico (Comte 1983). Por su parte, en las "Tesis sobre Feuerbach", Marx (1971) sostenía enfáticamente la relación dialéctica entre teoría y práctica. Weber (1988) rechazaba, a comienzos del siglo XX, la idea de la ciencia como una fría operación de cálculos realizados en laboratorio, repetición de fórmulas que se pierden en sistemáticas operaciones que no se sabe de dónde vinieron ni para donde van.

Es cierto que no es posible formar un investigador sin que este posea conocimientos de las metodologías específicas. Sin embargo, no resulta tan claro que enseñar metodología genere automáticamente la formación de investigadores o que enseñar metodologías no genere en los estudiantes, después que son 
aprobados en los cursos, una baja capacidad para utilizar en sus investigaciones futuras las herramientas aprendidas en los cursos.

Y aquí quizás sea interesante retomar nuestra cuestión inicial sobre la función de las asignaturas de metodología de la investigación. Dependiendo de nuestro posicionamiento en relación a ello, podemos discutir las estrategias de las enseñanzas de metodología, por lo menos, de dos formas distintas. Si lo que queremos es sencillamente transmitir metodologías, probablemente no tengamos grandes cosas a cambiar. Quizás podamos introducir en nuestras clases nuevas metodologías de la enseñanza; nuevas tecnologías de la información y comunicación o incluso podamos cambiar nuestra didáctica, adoptando formas que tornen a aquellas más interesantes para los alumnos, incluyendo una mayor democratización en la relación profesor-alumno.

No obstante, si como proponemos el objetivo de las asignaturas de metodología debería ser contribuir a la formación de investigadores sociales, además de cambios en los formatos de las clases, probablemente, tendríamos que cambiar la estructura de nuestras disciplinas y su relación con nuestro objeto de enseñanza y aprendizaje (las metodologías de las ciencias sociales), así como concebir relaciones diferentes a las actuales entre la enseñanza metodológica y las prácticas de investigación.

\section{Cambiar nuestra relación con las metodologías}

Colocar los puntos críticos en relación a la formación de investigadores sociales es mucho más sencillo a la hora de escribir que a la de practicar un cambio. Una cuestión que podría ingresar en la agenda académica es la de hasta dónde las universidades actuales están preparadas para estimular la formación de científicos sociales críticos (Santos 2008).

Para formar investigadores, potencializando las clases de metodología, es necesario, como primer paso, un cambio de mentalidad de los docentes y de los estudiantes en relación a la ciencia. Eso ya lo decía Wright Mills (1976), en su conocida apertura de la Imaginación Sociológica, cuando defendió la idea de la sociología como un elemento cotidiano en la vida de las personas, un campo de conocimiento que tiene entre sus vocaciones democratizar las reflexiones sobre las praxis sociales. Algo semejante viene argumentando Santos (1989) cuando defiende el quiebre de los formatos de los "paradigmas científicos tradicionales" -y esta es una de sus principales rupturas con el discurso de la modernidad-y la necesidad de la ciencia ser una apropiación del conjunto de la sociedad, así como un diálogo con otros tipos de conocimiento.

No obstante, cambiar mentalidades o aun paradigmas, desde la perspectiva de las asignaturas de metodología, representa un enorme desafío; en buena medida porque no solamente los docentes, sino también los estudiantes mantienen con las metodologías una relación muy semejante a la que pasa en los restaurantes donde, al entrar, esperamos el menú de la casa para elegir lo que vamos a consumir. Este es un problema observado por diferentes autores, como, por ejemplo, Bourdieu, Chamboredon y Passeron quienes afirman que: "A la tentativa que siempre surge de transformar los preceptos del método en recetas de cocina científica o en objetos de laboratorio, sólo puede oponérsele un ejercicio constante de vigilancia epistemológica que, subordinando el uso de técnicas y conceptos a un examen sobre las condiciones y los límites de su validez, proscriba la comodidad de una aplicación automática de procedimientos probados y señale que toda operación, no importa cuán rutinaria y repetida sea, debe repensarse a sí misma y en función del caso particular" (1994:16). 
Tal vez el paso propuesto por Boaventura de Sousa Santos en el sentido de la democratización de la ciencia sea gigantesco en el estado actual de nuestras disciplinas, sin embargo, cambiar la orientación de las metodologías, con el objetivo de que esta salte la valla representada por la transmisión monocorde de métodos y/o técnicas en cursos aislados y amurallados en los programas de estudio, pueda ser un primer paso para una formación más adecuada y crítica de los futuros investigadores sociales.

Lo dicho cobra una especial relevancia cuando observamos que en las diferentes asignaturas, que componen los programas de estudio de los cursos brasileños de Ciencias Sociales, es perceptible el espacio privilegiado (muchas veces el único) ocupado por el área metodológica para introducir los estudiantes en el mundo de la investigación (Villas Bôas 2003). Desconsiderar este aspecto no solamente reduce el potencial formador de las asignaturas metodológicas, sino también el de todo el proceso educacional de los futuros científicos sociales (4).

Si analizamos la estructura de los cursos de grado en Ciencias Sociales una primera cuestión que llama la atención es la separación entre la enseñanza de metodologías y las prácticas de investigación. Esto es especialmente importante en Brasil, donde las estructuras curriculares de los cursos de ciencias sociales tienden a prescribir una disociación entre asignaturas en las cuales se aprenden métodos de investigación generalmente existe la división de este contenido en dos o más asignaturas temáticas (por ejemplo, metodología 1 y 2)- y otras donde estos conocimientos son aplicados en el desarrollo de proyectos de investigación.

Otra separación adicional es la establecida entre asignaturas dedicadas, por un lado, a las metodologías cualitativas de la investigación y, por otro, a las cuantitativas. Esta es una cuestión que llama especialmente la atención porque hasta poco tiempo atrás existió una identificación de la relación entre lo cualitativo y lo cuantitativo como una contraposición entre ciencias sociales críticas y conservadoras. La vigencia de esta separación puede ser consecuencia de la identificación -existente hasta poco tiempo atrás- de lo cualitativo y lo cuantitativo como una contraposición entre métodos y técnicas cualitativas y cuantitativas en términos de paradigmas que no conversan entre sí, esto es, entre los que existiría un alto grado de "inconmensurabilidad", en una forma de razonamiento vinculada, pensamos de forma errónea, al modelo de análisis de ciencia de Khun (1971).

No tenemos datos que comprueben el grado de persistencia de una división "paradigmática" entre metodologías cuantitativas y cualitativas, como ya existió antes y generó fuertes debates, por lo menos, hasta bien entrados los años ochenta del siglo pasado (Errandonea y Supervielle 1986). Respecto a eso no basta con realizar una declaración de complementariedad o de relaciones de amistad entre unas y otras metodologías, cuando en la práctica de enseñanza se las aísla. Tal separación acaba estimulando una enseñanza segmentada de la metodología en la cual los diferentes métodos y/o técnicas de investigación son presentados de forma abstracta, como cuerpos extraños y muchas veces con objetivos contrapuestos (por ejemplo, entre otros, el que sostiene que las técnicas cualitativas estarían orientadas al contexto de descubrimiento y las técnicas cuantitativas al contexto de justificación) de investigación social.

Wacquant defiende, en una solución más adecuada para esta cuestión, la importancia de un "politeísmo metodológico" (1995:30), por el cual no hay, por un lado, una fetichización de un determinado método o técnica, y por el otro, se pregona una reflexión orientada para que exista una coherencia entre las formulaciones teóricas y los procedimientos metodológicos adoptados, así como de estos últimos entre sí. Un autor de la talla de Bourdieu defendía de la siguiente manera la utilización de diferentes métodos de 
aproximación a la realidad: "es preciso desconfiar de los rechazos sectarios que se esconden por detrás de las profesiones de fe demasiado exclusivas y tentar, en cada caso, movilizar todas las técnicas que, dada la definición del objeto, puedan parecer pertinentes y que, dadas condiciones prácticas de recolección de los datos son prácticamente utilizables" (Bourdieu 2000:26).

Esta complementariedad de métodos y técnicas ha sido reivindicada, ampliamente, desde diferentes posiciones en los últimos años, dejando para atrás debates como los anteriores (Cea D’Ancona 1998:43-62).

Tal vez pueda conjeturarse que las fronteras creadas (a pesar de las buenas intenciones) tengan su origen en la formación de los propios docentes, que especializados en algunas de las metodologías mantienen dificultades para exponer a los estudiantes las integraciones posibles a ser realizadas entre ellas. En el mejor de los casos los alumnos y alumnas más sobresalientes llegan a memorizar aspectos considerados relevantes de las metodologías, mas acaban entendiendo de forma precaria las implicaciones de su utilización.

Por fin, existe una escasa integración entre las asignaturas de metodología y las investigaciones desarrolladas por los docentes de los cursos, estimulando una división entre lo que se enseña en clase y las prácticas reales de investigación llevadas adelante en los departamentos y cursos.

La idea de explicitar didácticamente nuestras experiencias de investigación nos es presentada de forma anecdótica por Galtung (1995), quien en cierta oportunidad, estando detenido en Bogotá y sin poder desplazarse rápidamente hacia Caracas, debido a que el próximo avión partiría en algunos días, le fue sugerido viajar hacia Nueva York o hacía París y luego hacía Caracas. Conforme cuenta el autor, en un sencillo problema de desplazamiento le fue evidente como nunca antes el peso que tenían en el sistema mundial determinados países, esto es, como la "interacción [se da] entre los grandes".

Algo semejante se puede ver en el relato de Robertt (2007) que cuenta que una de las hipótesis principales de su tesis doctoral, sobre el espacio social de una empresa, surgió al final de una reunión de trabajadores sindicalizados en la cual uno de estos realizó la "confidencia", fuera de toda situación formal de entrevista, de que en esa organización habían dos grandes líneas gerenciales, una más "contable" con la cual era muy difícil de relacionarse y otra más "humanista" que procuraba el diálogo. A partir de esa "idea" inicial se construyó una hipótesis de mayor complejidad que colocaba en juego la forma en que en la organización en cuestión se llevaba adelante todo un proceso de reorganización empresarial.

Volviendo a nuestro centro de interés, la articulación de la enseñanza de metodologías con la discusión sobre los proyectos de investigación y el desarrollo del trabajo de campo de los docentes podría contribuir al enriquecimiento del proceso de formación investigativa y al fortalecimiento de la idea de la investigación como una acción colectiva, esto es, como un trabajo desarrollado, no apenas por investigadores aislados, sino por grupos.

\section{Articular metodologías y praxis de la investigación}

Delante de las cuestiones tratadas en este artículo, pueden ser planteados algunos dilemas: ¿hasta qué punto enseñamos en las asignaturas de metodología aspectos que tocan solamente tangencialmente el mundo práctico de la investigación social? E, inversamente, ¿cuánta de la riqueza generada en las prácticas de investigación llevadas adelante por los profesores-investigadores termina no siendo socializada en la formación de los estudiantes-futuros investigadores? 
Sobre este último aspecto, se coloca la incertidumbre sobre el hecho de las investigaciones realizadas en las universidades en constituirse en verdaderas "zonas desconocidas", no accesibles en términos de "recetas" prácticas a los estudiantes. Esto puede deberse tanto por un posible cuestionamiento a la legitimidad de los científicos sociales (abrir sus investigaciones puede ser percibido como una apertura a la impugnación, entre otros aspectos, de sus procedimientos de obtención de la "prueba"), como a una formación académica que no visualiza en los productos del trabajo científico un objeto de examen de los alumnos (5). Paradójicamente este último aspecto coloca en cuestión dos pilares centrales de la construcción de la ciencia moderna: por un lado, el de que toda experiencia científica debe de estar disponible a cualquier miembro de la comunidad, de forma de garantizar el cotejo de los resultados a los cuales se llegó; y, por otro, el de la comunicabilidad.

Con el objetivo de indicar orientaciones sobre los procedimientos y avatares de la investigación científica, incluyendo "pormenores aparentemente ínfimos, inclusive insignificantes" (Bourdieu 2000:27), esto es, aquellos aspectos que acostumbran quedar ocultos en los informes metodológicos y que hacen a la propia construcción del objeto sociológico, varios asuntos merecen nuestra atención. Entre ellos, se encuentran el problema de cómo se procesan las tomas de decisión fundamentales en el curso de una investigación, qué tipos de muestra construimos con frecuencia (si es que utilizamos este procedimiento estadístico) y hasta dónde se acercan o se alejan de las muestras ideales presentadas en los manuales estadísticos. No menos importantes son los cuestionamientos sobre cómo surgen nuevas ideas e hipótesis en el transcurso de una investigación, así como los problemas reales que enfrentamos para acceder a grupos y a organizaciones sociales y cómo se procesa la negociación de ese acceso (los manuales de metodología acostumbran colocar el acceso como una etapa primera de la investigación, que aunque problemática, una vez resuelta no precisa volver a ser planteada). Dependiendo de la organización o grupo social y muchas veces de los conflictos presentes en el espacio social que se está analizando, el acceso a la información puede ser facilitado o no, lo que implica en discutir las condiciones del acceso y la manera como construimos la confianza con nuestros "objetos de estudio" (6).

También en términos de la construcción de las bases de datos muchas veces es necesario que el investigador sepa decidir qué hacer ante un dato empírico que no se comporta de acuerdo con lo esperado teóricamente, o en consonancia con nuestro diseño de investigación, y la necesidad de conciliar resultados contradictorios de tales bases, para no forzar al dato a decir lo que no puede decir.

Por último, pero no menos importante, queda la pregunta que desde los orígenes de la ciencia social genera ansiedad en los investigadores: ¿qué estrategias utilizamos para descubrir el efecto de nuestras concepciones del mundo y de nuestros valores sobre el material empírico, alejados de la ilusión positivista de separación rígida entre sujeto y objeto?

Aquí no se trata de establecer normativamente una forma única de construir un objeto sociológico, sino de explicitar decisiones metodológicas y prácticas de investigación que pueden resultar pertinentes en determinadas circunstancias. Se trata de presentar el "habitus científico" (Bourdieu 2000:23) en funcionamiento o, lo que es lo mismo, rescatar la inmensa cantidad de "metodologías desperdiciadas" expresión inspirada en el concepto de "experiencia desperdiciada" de Santos (2006)- que tienen por origen nuestras prácticas de investigación.

Uno de los principales problemas en la enseñanza de metodologías de las ciencias sociales es la excesiva abstracción del método que, en muchos manuales de investigación, es presentado como una aglomeración 
de técnicas despegadas de la realidad. Esto representa un proceso de reificación del mundo académico en el cual los investigadores sociales son presentados como agentes que analizan realidades al modo de piezas que deben ser encajadas en un puzle.

Desde una perspectiva crítica, superar esta situación requiere un retorno a la praxis de la investigación, enfrentada como un proceso de constante reconversión analítica. Y acá son sugerentes los conocidos versos de Antonio Machado: “Caminante, son tus huellas el camino y nada más; caminante, no hay camino, se hace camino al andar".

El simple hecho de reposicionar las metodologías alrededor de la problemática del análisis de las experiencias científicas representa un importante avance en lo que relacionado a la formación de investigadores en la universidad. Esto significa, como afirma Melucci (2005), una desacralización de la ciencia, la cual pasa a ser analizada como una práctica social más. No obstante, las ciencias sociales representan una práctica especial, principalmente cuando llevan en consideración la lección hermenéutica de que su objeto de análisis no es externo, requiriendo del científico un constante ejercicio de reflexividad como hilo conductor del análisis de las relaciones sociales, no apenas desde una perspectiva aislada sino en constante diálogo con la teoría. En otras palabras, existe un relacionamiento permanente entre la investigación y la teoría social. Para nosotros, como investigadores, la cuestión no es solamente lo que producimos, sino cómo lo hacemos, siendo esto inseparable en el proceso de la investigación" (May 2004:44).

\section{Comentarios Finales}

Para terminar cabe rescatar positivamente los esfuerzos docentes consientes (muchas veces titánicos) para transmitir las diferentes técnicas de investigación utilizadas por los sociólogos, los debates académicos en muchas universidades sobre estas cuestiones, así como los seminarios de investigación (principalmente a nivel de postgrados) en los cuales se debaten ampliamente los procesos concretos de investigación desarrollados por los alumnos. Con ser aspectos positivos, resultan insuficientes, ya que mucho camino aún se puede andar.

En este texto hemos procurado ofrecer algunas indicaciones en una nueva dirección tales como: integración y discusión de las prácticas de investigación de profesores y alumnos en los salones de clase, incluyendo los pormenores generalmente considerados insignificantes de la labor científica; programas de estudio que integren en forma más adecuada sus disciplinas, tanto las teorías con las metodologías como estas últimas con las disciplinas que involucran prácticas de investigación; seminarios en que investigadores "cuenten" sus investigaciones, principalmente los procedimientos prácticos como por ejemplo el de "no perder una idea" o el de obtener y mantener un "acceso"; una mayor participación de los estudiantes en las investigaciones de los profesores, como por ejemplo es el caso de las becas de iniciación científica en las universidades brasileñas (otras formas pueden pensarse que no requieran de un involucramiento institucional del estudiante y que lo pongan, aunque sea de forma intermitente, en contacto con el universo de la investigación científica), y la creación de puentes entre nuestras disciplinas para construir investigadores que posean creatividad para generar nuevas ideas sobre el mundo social y desenvuelvan formas sistemáticas y rigorosas para someterlas a prueba. En suma, se trata del aprendizaje que resulta del enfrentamiento con las rebeldías de la práctica, las cuales no se dejan aprisionar obedientemente a los artefactos construidos en el laboratorio sociológico. 


\section{Notas}

(1) Estas cuestiones impulsaron un debate que fue central en nuestro campo de conocimiento en el siglo XX, articulando las más importantes corrientes de pensamiento como positivismo, marxismo, funcionalismo, postmodernismo, entre otras.

(2) La variedad encontrada es imposible de ser registrada en un artículo de esta naturaleza, por lo que la generalización aquí planteada debe considerarse como una aproximación más que una demostración empírica del funcionamiento de los diferentes programas de estudio.

(3) Moraes (2003) muestra como son organizados los currículos en los Cursos de Ciencias Sociales en Brasil.

(4) Cabría subrayar que aun existiendo un debate muy rico en algunas universidades brasileñas sobre la enseñanza de la metodología, en la mayor parte del país hay poca preocupación con tales cuestiones.

(5) Los obstáculos creados, lentamente superados por el mundo científico, para la difusión de sus productos, por ejemplo el acceso irrestricto a teses completas de autores, puede representar otra faz de este tema.

(6) Por ejemplo, ¿qué alternativas buscamos cuando agentes o actores que ocupan posiciones estratégicas, en el espacio social que estamos estudiando, se recusan por diferentes motivos (tales como la posesión de diferentes capitales cultural, económico u otros de los del investigador o la aprensión con la divulgación de determinadas informaciones) a ser entrevistados?

\section{Bibliografía}

Bastos, É. R. (ed) 2006. Conversas com sociólogos brasileiros. São Paulo: Ed. 34.

Betancourt, W. et al. 1990. La enseñanza, la reflexión y la investigación filosófica en América Latina. Madrid: Tecnos.

Bomeny, H. y Birman, P. (eds) 1991. As assim chamadas ciências sociais: formação do cientista social no Brasil. Rio de Janeiro: UERJ.

Bourdieu, P. 2000. O poder simbólico. Rio de Janeiro: Bertrand Brasil.

Bourdieu, P., Chamboredon, J-C y Passeron, J-C. 1994. El oficio del sociólogo. Madrid: Siglo XXI.

Bourdieu, P. y Passeron, J-C. 1982. A reprodução. Rio de Janeiro: Francisco Alves.

Cardoso, R. 1986. A aventura antropológica. Rio de Janeiro: Paz e Terra.

Cea D’Ancona, M. Á. 1998. Metodología cuantitativa. Estrategias y técnicas de investigación social. Madrid: Síntesis.

Comte, A. 1983. Curso de filosofia positiva. São Paulo: Coleção Os Pensadores.

Errandonea, A y Supervielle. M. 1986. El lugar de las técnicas cualitativas. Revista de Ciencias Sociales 1: 5970. 
Freire, P. 1981. Pedagogía do oprimido. Rio de Janeiro: Paz e Terra.

Khun, T. 1971. La estructura de las revoluciones científicas. México: Fondo de Cultura Económica.

Galtung, J. 1995. Investigaciones teóricas. Sociedad y cultura contemporáneas. Madrid: Tecnos.

May, T. 2004. Pesquisa social. Porto Alegre: ArtMed.

Melucci, A. 2005. Por uma sociología reflexiva. Petrópolis: Vozes.

Marx, K. y Engels, F. 1971. La ideología alemana. Montevideo: Pueblos Unidos.

Moraes, A. C. 2003. Licenciatura em Ciências Sociais e Sociologia. Tempo Social 15(1): 5-20.

Pessanha, E. y Villas Bôas, G. (eds). 1995. Ciências sociais: ensino e pesquisa na graduação. Rio de Janeiro: J. Editora.

Reis, E.P., Reis, F.W. y Velho, G. 1997. As ciências sociais nos últimos 20 anos: três perspectivas. Revista Brasileira de Ciências Sociais 15(35).

Robertt, P. 2007. La construcción metodológica de un campo de luchas en una organización empresarial. Estudio de caso en una empresa industrial uruguaya. XXVI Congreso de la Asociación Latinoamericana de Sociología. Guadalajara.

Santos, Boaventura de Sousa. 2008. A Universidade no século XXI. Coimbra: Almedina.

Santos, Boaventura de Sousa. 2006. A crítica da razão indolente. São Paulo: Cortez.

Santos, Boaventura de Sousa. 1989. Uma introdução a uma ciência pós-moderna. Rio de Janeiro: Graal.

Villas Bôas, G. 2003. Currículo, iniciação científica e evasão de estudantes em Ciências Sociais. Tempo Social 15(1): 45-62.

Wacquant, L. J. D. 1995. Introducción. En: Bourdieu, P; Wacquant, L. J. D. Respuestas por una antropología reflexiva. México, DF: Grijalbo, pp. 1-30.

Weber, M. 1988. El político y el científico. Madrid: Alianza.

Willis, P. 1988. Aprendiendo a trabajar. Como los chicos de la clase obrera consiguen trabajos de la clase obrera. Madri: Akal.

Wright Mills, C. 1976. A imaginação sociológica. Rio de Janeiro: Zahar.

Recibido el 30 Sep 2009

Aceptado el 25 Jun 2010 